\title{
Enseñar Ingeniería, ¿para qué? Una lectura desde el pensamiento de Amartya Sen y Martha Nussbaum
}

\author{
Maria Alejandra Bosio ${ }^{1}$ \\ Universidad Católica de Córdoba \\ Agustina Brizuela ${ }^{2}$ \\ Pontificia Universidad Católica Argentina \\ Horacio Jose Brizuela ${ }^{3}$ \\ Pontificia Universidad Católica Argentina \\ Gustavo Alberto Chiodi ${ }^{4}$ \\ Universidad Católica de Córdoba
}

\section{Ensayo}

Material original autorizado para su primera publicación en el Journal de Ciencias Sociales, Revista Académica de la Facultad de Ciencias Sociales de la Universidad de Palermo.

Recepción: 16-10-2019

Aceptación: 30-03-2020

Resumen: Este ensayo se propone reflexionar acerca de los desafíos que presenta a la enseñanza de la Ingeniería el actual contexto internacional, especialmente el latinoamericano, donde se observan grandes desigualdades socioeconómicas, brechas tecnológicas respecto a países más sofisticados, falta de inclusión y sensibilidad hacia los problemas más extremos. Asimismo, el contexto global es desafiante, desde la exacerbada competencia material entre países poderosos, problemas ambientales y amenazas climáticas. Los Estados democráticos afrontan primordialmente los flagelos de las diferencias de desarrollo y el problema de la desigualdad. Surge la pregunta acerca de si la tecnología ha promovido, hasta ahora, el desarrollo inclusivo o no. ¿Ha expandido las brechas tecno-

\footnotetext{
${ }^{1}$ Profesora titular de la Universidad Católica de Córdoba. Investigadora.

Correo electrónico: malejandra.bosio@gmail.com

2 Estudiante de Doctorado en Ciencias Políticas (UCA), Lic. en Relaciones Internacionales (UNC). Asesora en la Secretaría de Innovación Pública, perteneciente a la Jefatura de Gabinete de Ministros de la Nación. Experta Asociada en Transformación Digital e Innovación Urbana de la red internacional Cities Forum. Correo electrónico: agustina.brizuela@gmail.com

${ }^{3}$ Estudiante de Doctorado en Ciencias Políticas (UCA), MBA (Universidad de Bologna), Lic. en Física (UNC). Correo electrónico: horacio.brizuela@gmail.com

${ }^{4}$ Profesor titular en la Facultad de Ingeniería, Universidad Católica de Córdoba.

Correo electrónico: gchiodi@gmail.com
} 
económicas 'inter' e infra-nacionales? Si así fuera, ¿puede la Ingeniería, por ejemplo, constituirse en una profesión que minimice las desigualdades e impulse un mayor acceso a oportunidades equitativas?, ¿puede llegar de modo cada vez más ubicuo y capilar a una base más amplia de beneficiarios, para el acceso ecuánime al conocimiento? Se considera que la Ingeniería tiene un nuevo rol en el mundo actual. Para abordar este paradigma, el presente trabajo recupera el concepto de desarrollo del Premio Nobel de Economía Amartya Sen. Se analiza su teoría del desarrollo basado en las capacidades, la libertad y la consecuente cooperación, con impacto en nuevos abordajes de la Educación, en particular de la educación en Ingeniería. Se descubre un nuevo ‘¿para qué?' de esta profesión, encontrando en el pensamiento de Sen una nueva y muy noble vocación para la misma. Por último, se propone un análisis de un programa educativo en Ingeniería de la Universidad Católica de Córdoba desde el enfoque de las capacidades, con el objetivo de identificar en la práctica modos de poner en marcha este nuevo rol de la Ingeniería partiendo desde la educación para la libertad.

Palabras clave: Ingeniería; capacidades; libertad; desarrollo inclusivo.

\section{Teaching Engineering, for what? An approach from Amartya Sen and Martha Nussbaum's thought}

Abstract: In this essay we intend to reflect on the challenges that the current international context presents to the Engineering education, especially in the Latin American background, where there are great socioeconomic inequalities, technological gaps regarding more sophisticated countries, lack of inclusion and sensitivity towards more extreme problems. Likewise, the global context is challenging due to the exacerbated material competition between powerful countries, environmental problems and climate threats. Democratic States primarily face the development gaps and the problem of inequality. We wonder if, so far, technology has promoted inclusive development or not. Has technology contributed to expand the techno-economic gap between nations and within them? If so, can Engineering, for example, become a profession that minimizes inequalities and support greater access to equitable opportunities? Can it reach an increasingly ubiquitous and capillary way to a wider base of beneficiaries and to equitable access to knowledge? We believe that Engineering has a new role in today's world. To address this paradigm, we rely on the concept of development proposed by the Nobel Prize in Economics, Amartya Sen. We analyze his theory of development based on capabilities, freedom and consequent cooperation, with an impact on new approaches to Education, in particular Engineering Education. We discover a new one purpose for this profession, finding in Sen's thought a new and very noble vocation for it. Finally, we propose an analysis of an educational program in Engineering carried out by the Catholic University of Córdoba from the capabilities approach. It's aim is to identify, in practice, 
some novel strategies to implement this new role of Engineering on the base of education for freedom.

Keywords: Engineering; capabilities; freedom; inclusive development.

"Focusing in freedom does indeed make a difference"

- Amartya Sen

\section{Introducción}

\subsection{Motivación y delimitación del campo de estudio}

Este ensayo se propone analizar la posibilidad de descubrir un nuevo rol de la Ingeniería, a partir de preguntas por la coyuntura latinoamericana y global del presente.

Acemoğlu y Robinson (2012) se preguntan por las enormes diferencias en los ingresos y parámetros de bienestar que dividen al mundo. Su afirmación principal es que en diversas naciones se generan espirales virtuosas y espirales viciosas en economías de mercado. En las situaciones virtuosas las personas pueden 'comprar' mejores estándares de vida, tales como 'coberturas de salud', entre otros bienes o servicios. El contraste entre ambos casos es debido, según Acemoğlu y Robinson (2012), a que el crecimiento económico y la prosperidad están asociados con instituciones económicas y políticas de tipo inclusivo, en contraposición a las instituciones 'extractivas' que dan lugar a estancamiento y pobreza. Este fenómeno sucede desde la América Latina colonial, en la cual algunos consiguieron ejercer el poder y crearon instituciones económicas para enriquecerse e incrementar su propio poder en detrimento de otros sectores sociales.

Otro factor crucial para el desarrollo es el conocimiento tecnológico y la innovación, junto a los grados de complejidad con que esta se logra en los diversos países: algunos más simples, otros más sofisticados. Acemoğlu y Robinson (2012), al analizar el subdesarrollo, hacen hincapié en la falta de 'innovación destructiva propia', la cual consiste en el constante reemplazo de tecnologías y formas de trabajo por otras (aparentemente) más 'avanzadas' (lo cual beneficiaría el desarrollo autónomo, sin dependencias extranjeras). En este punto surge una primera pregunta referida al rol de la Ingeniería: ¿es ella motor de conocimiento innovador que llega a toda la base poblacional para ser partícipe de instituciones económicas inclusivas?, o, por motivos de desigualdad de oportunidades, ¿queda ella limitada a un sector de la población, el que logra la capacidad de los conceptos abstractos y el pensamiento lógico-matemático que requiere?

${ }^{5}$ Amartya Sen (2000, p. 354). 


\subsection{Desigualdades en América Latina en}

El Padre de la Ciencia Política argentina, Guillermo O’Donnell (2010, 2011), estudió las relaciones entre las democracias latinoamericanas y los problemas de desarrollo y desigualdad. Lo hizo para precisos casos de análisis en determinados períodos temporales. No obstante, ciertas características de sus conclusiones son válidas porque se han repetido situaciones empíricas similares. En esencia, O'Donnel demuestra contundentemente que no es cierta la 'ecuación optimista' (a mayor desarrollo socioeconómico, mayor posibilidad de salud democrática, como si entre estos dos factores hubiera una función monótona creciente) de otros autores.

Un problema crucial que O'Donnell $(2010,2011)$ identificó para el escenario latinoamericano de mitad de siglo XX fue que, no obstante períodos de prosperidad por agroexportaciones, surgió un indeseable antagonismo interno entre sectores que promovieron la industrialización y el sector conservador agroexportador. Esta rivalidad es en cierto modo el origen de fracturas perdurables, una ruptura honda en las sociedades de la región. El problema generó una 'dificultad endógena' a la industrialización, al desarrollo tecnológico y la innovación permanente, dado que interrumpió el desarrollo tecnológico y llevó a una falta de estrategia industrial integral intensiva. La situación condujo a la falta de desarrollo de conocimiento tecnológico del nivel de sofisticación que se estaba produciendo en otros países más 'avanzados'.

\subsection{La globalización, el desarrollo y la pregunta por la Ingeniería}

El contexto actual ha cambiado profundamente desde la década de 1980, fecha de nacimiento de la globalización, en la que tanto la desregulación de los mercados financieros, el comercio internacional y el nacimiento de la Web dieron lugar a un formato social radicalmente diferente. El mundo entero adopta tecnologías digitales y los países compiten mediante la innovación, la tecnología y el conocimiento.

Ante la paradójica dicotomía latinoamericana arriba mencionada: la innovación tecnológica como motor de desarrollo económico y el antagonismo (egoísta) entre sectores sociales, surge una fundamental pregunta. ¿Para qué la Ingeniería? ¿Sólo para desarrollos que oscilan según los factores humanos que dividen a la sociedad? ¿Puede ser asociada la Ingeniería con el desarrollo integral humano que abra caminos inclusivos? ¿Qué elecciones de diseño pedagógico de la Ingeniería pueden abrir estos caminos? ¿Qué libertades son necesarias para disfrutar de tales opciones?

Se abordarán las preguntas planteadas desde los autores que han investigado en gran profundidad las raíces del genuino desarrollo, superando diferencias de todo tipo 
ideológico, y han humanizado tanto la economía como las políticas públicas para la Educación y todo tipo de sano avance humano: el Premio Nobel Amartya Sen y la reconocida filósofa política Martha Nussbaum.

Para esta tarea, se enunciará la teoría de las capacidades de Amartya Sen, su relación con la libertad y el desarrollo. Se analizará el desarrollo como una amplia posibilidad humana más que una situación medible por variables macroeconómicas. Se propondrán conclusiones acerca de cómo el enfoque en capacidades provee un nuevo 'para qué' a la tecnología y a la Ingeniería en particular. Desde allí, será defendido el rol fundamental que posee la Educación en Ingeniería para la expansión del desarrollo humano en tanto su objetivo es formar individuos que, en primer lugar, dispongan de las libertades y oportunidades necesarias para llevar a cabo la vida que tienen razones para valorar y, en segundo lugar, pongan en práctica la promoción y expansión del desarrollo para todos. Específicamente, con foco en encaminar la reflexión teórica a un ejercicio práctico de observación y propuesta, se intentará un diálogo entre las nociones analizadas y una realidad educativa específica de nuestra región: el programa educativo de la carrera de Ingeniería en Computación de la Universidad Católica de Córdoba. Serán analizadas en la práctica algunas estrategias mediante las cuales se observa que se pone en marcha la educación para la libertad en la enseñanza de la Ingeniería con ánimos de abrir interrogantes para futuras investigaciones impulsadas por la búsqueda de este nuevo ¿para qué? a la Ingeniería en Computación.

\section{Argumentación teórica}

\subsection{El desarrollo como capacidades y libertad}

Según Sen (2000), el desarrollo puede considerarse, "como un proceso de expansión de las libertades reales de las que disfruta la gente" (p. 17). Este enfoque difiere radicalmente de otras "visiones más limitadas del desarrollo, como identificar el desarrollo con el crecimiento del producto nacional bruto, o con el aumento de los ingresos personales, o con la industrialización, o con el avance tecnológico, o con la modernización social" (Sen, 2000, p.17). Estos criterios no son negados por el autor, pero son superados por un enfoque integral de las libertades, dado que "las libertades dependen también de otros determinantes, como los arreglos sociales y económicos (por ejemplo, instalaciones para la educación y la atención médica), así como los derechos políticos y civiles" (Sen, 2000, p.17). Dado que la libertad es lo que genera el genuino desarrollo, entonces es fundamental focalizarse en el objetivo general, en lugar de en los medios o recursos particulares. Así, "ver el desarrollo en términos de expansión de las libertades sustantivas dirige la atención a los fines que hacen que el desarrollo sea importante, en lugar de limitarse a algunos de los medios" (Sen, 2000, p. 17). 
Para Sen, la libertad es crucial en el proceso de desarrollo por dos principales motivos: en primer lugar, por el motivo "evaluativo" dado que "la evaluación del progreso debe hacerse principalmente en términos de si se mejoran las libertades que tienen las personas" (Sen, 2000 , p.18); en segundo lugar, por el motivo de la efectividad, dado que "el logro del desarrollo depende completamente de la acción en cuanto agencia libre de las personas" (Sen, 2000, p. 18).

Más aún,

La agencia libre y sostenible emerge como un importante motor de desarrollo. La agencia libre no solo es una parte 'constitutiva' del desarrollo, sino que también contribuye al fortalecimiento de las agencias libres de otros tipos. Las conexiones empíricas que se exploran ampliamente en este estudio vinculan los dos aspectos de la idea de 'desarrollo como libertad' (Sen, 2000, p.18).

Ahora bien, ¿de qué fundamentos proviene la libertad? Urquijo (2014) describe nítidamente el fundamento de la libertad según Sen, partiendo del análisis de los funcionamientos y las capacidades.

Lo que importa para evaluar el bienestar no es lo que una persona tiene, como podrían ser los bienes de consumo y la apropiación de las propiedades de estos bienes, sino lo que consigue realizar con lo que tiene. Las características de los bienes no dicen nada de lo que la persona puede hacer con esas propiedades (Urquijo, 2014, p.7).

Urquijo (2014) explica que la teoría de Sen plantea el modelo de agencia asignándole cadenas de funcionamientos que se representan en un espacio vectorial. Estas cadenas funcionales son los logros de la persona. En cambio, las capacidades, que se representan en el mismo espacio vectorial, son conjuntos de dichos logros. "Una capacidad es un conjunto de vectores de funcionamientos o una suma de vectores de estos funcionamientos, que reflejan la libertad de la persona para alcanzar aquello que valora" (Urquijo, 2014, p.10). Así, "la capacidad proporciona un punto de vista desde el que valorar las condiciones sociales, políticas y económicas que viven las personas al interior de la sociedad" (Urquijo, 2014, p.10). Siguiendo con ello, Urquijo (2014) señala que "la combinación de funcionamientos refleja la capacidad y los logros reales. Los funcionamientos, entonces, alimentan las capacidades" (p.12). La falta de un funcionamiento es una señal de que una capacidad ha sido vulnerada, porque la misma requiere una cadena funcional de funcionamientos. Por ende, una capacidad se juzga, en función de los funcionamientos (Nussbaum, 2002 en Urquijo, 2014). Así, "una combinación de funcionamientos es un punto en ese ámbito, mientras que la capacidad es un conjunto de tales puntos" (Sen, 1992, p.50). 
Ahora bien, ¿dónde se encuentra el salto hacia la libertad? Urquijo (2014) nos lo aclara:

(...) La realización de tales logros es lo que constituye la capacidad de una persona. La capacidad, que es un conjunto de $\mathrm{n}$ funcionamientos alcanzados, refleja la libertad de la persona, una libertad para hacer esto o aquello. La libertad se constituye como un aspecto de relevancia moral y política, entre otras cosas, porque si podemos vivir sin deficiencias nutricionales, con cobijo y gozar de buena salud, teniendo la posibilidad de hacer un uso público de la razón, de participar en los ámbitos políticos, sociales y económicos en mi comunidad, entre otros funcionamientos; se expresa, entonces, una libertad como capacidad que refleja la oportunidad real para alcanzar aquello que valoro (p.12).

Se concluye, entonces, que el conjunto de capacidades representa la libertad para lograr los resultados de las opciones de los funcionamientos, es decir, las distintas combinaciones de funcionamientos que se pueden realizar, es decir "El conjunto de capacidades representa la libertad general de una persona para alcanzar aquello que valora" (Urquijo, 2014, p.12). La conclusión de Urquijo (2014) es, entonces, "que el foco para Sen son las habilidades realizadas, representadas en lo que una persona es capaz de hacer realmente, es decir, sus logros. O el conjunto de capacidades expresado en sus oportunidades reales" (p.13). Según la teoría de las capacidades, tanto los funcionamientos como las capacidades entregan diversas clases de información: "los funcionamientos sobre las cosas que hace una persona y las capacidades sobre las cosas que tiene libertad para hacer" (Sen, 2000, p.75).

La evaluación de un conjunto de capacidades se basa (primero) en la valoración de alguno o algunos de estos funcionamientos elegidos de este conjunto. Luego la obtención del bienestar de una persona puede ser valorada sobre la base del conjunto de capacidades, gracias a la identificación del valor de los funcionamientos logrados por la persona que se encuentran contenidos en el conjunto de capacidades seleccionadas (Urquijo, 2014, p.12).

Urquijo (2014) se pregunta, siguiendo a Sen, “¿por qué se debe tratar de ampliar esta base de información?" (p.14):

Cuando se evalúa el bienestar de una persona desde el conjunto de capacidades en vez de un funcionamiento elegido no hay ninguna pérdida informativa, porque el con- junto de capacidades contiene una serie de funcionamientos. Pero el argumento que lleva a ampliar la atención de los funcionamientos al conjunto de capacidades es porque presenta una mayor ventaja para conocer el estado real de una persona (Urquijo, 2014, p.14).

Se identifica aquí un rol preponderante de la Educación: favorecer la garantía de los funcionamientos básicos, construir sanos funcionamientos complejos, favorecer la activación 
de capacidades y amplias posibilidades de opción sobre la base informacional más amplia de la persona. He ahí la base de la libertad para que la persona no sólo goce de los bienes y logre con ellos resultados para sí, sino que también logre desarrollar, en libertad, opciones por el nosotros que habita en la esfera púbica: lo social, lo sanamente político y el desarrollo de todos. Una persona así completa no puede ser indiferente al déficit de desarrollo de los demás, y será partícipe de innovaciones que favorecerán a los más vulnerables, a los pudientes indiferentes, y, en fin, proveerá la facilitación de mejores equilibrios sociales, es decir, trabajará en pos de la equidad. Así, se pone en evidencia la posibilidad de profesiones hoy cruciales para el desarrollo de las naciones, como la Ingeniería, poniéndose al servicio de la innovación destructiva (en el sentido de reemplazar tecnologías y modos de trabajo por nuevos paradigmas), dotada de la gran libertad de expandirse también como motor de equidad social y desarrollo integral humano.

\subsection{La educación universitaria para la libertad y el compromiso}

Desde el enfoque propuesto por Amartya Sen (2000), se entiende a la educación como una libertad instrumental, entendiendo que la misma es un canal o medio para la expansión del desarrollo. Como se detalló anteriormente, el rol instrumental de algunos tipos de libertades consiste en que las mismas contribuyen a la expansión de la libertad humana entendida de forma general y, con ello, al desarrollo en términos de ampliación de las capacidades que los individuos tienen para perseguir el tipo de vida que tienen razones para valorar (Sen, 2000; 2012). Dentro de los diversos tipos de libertades instrumentales, Amartya Sen (2000) ubica la educación dentro del conjunto de oportunidades sociales, que influyen en la libertad sustantiva del individuo de vivir mejor. En relación con las oportunidades sociales, el economista indica que son relevantes no solamente respecto a las capacidades relativas a la vida privada, sino que las mismas también están interconectadas con otros tipos de libertades, como la mejor y más efectiva participación en actividades económicas y políticas.

Así entendida, la educación es una libertad instrumental fundamental para el desarrollo de un individuo. Si bien esta noción es de gran valor teórico y práctico en sí misma, realizando una lectura más precisa, profunda y exigente del enfoque de las capacidades, se comprende que la educación posee un rol instrumental en la expansión del desarrollo, más amplio y superador de la concepción anteriormente detallada. Para ello, se recupera el concepto de agencia propuesto por Amartya Sen (2000), quien se aleja de la concepción de gran parte de la literatura económica del agente como quien actúa en función de otro actor, denominado principal, y cuyos logros serán evaluados en términos de los objetivos de este último. Se entiende el concepto de agente propuesto por Sen (2000) como un miembro de la 
comunidad y como participante en actividades económicas, sociales y políticas. Éste actúa y produce cambios, que pueden ser juzgados en función de sus propios objetivos y valores (2000). Las libertades entendidas de forma instrumental son determinantes de la iniciativa individual: la expansión de la libertad impulsa la habilidad de las personas para desarrollarse a sí mismos y, además, para generar impactos y cambios en el mundo, lo cual resulta central para Amartya Sen (2000). Siguiendo con ello, Sen (2000) realza el fuerte vínculo que existe entre la libertad individual y el compromiso social. Alejándose de una larga tradición de la filosofía política moderna que escinde la libertad individual de la sociedad, el economista defiende la relación de interdependencia entre la libertad y la responsabilidad. Como seres reflexivos, tenemos la capacidad de contemplar las vidas de otros y afligirnos por las miserias que ocurren alrededor nuestro. Como miembros de una comunidad, no podemos escapar de la evidencia de que tales miserias son, también, nuestros problemas y nuestra responsabilidad y que está a nuestro alcance ayudar a remediarlas. Amartya Sen (2000) sostiene que negar ese llamado a nuestra atención sería equivalente a eludir una cuestión central de nuestra existencia social y señala la necesidad de reconocer la relevancia de nuestra humanidad compartida en las elecciones a las que nos enfrentamos. Con ello, se considera necesario recuperar aquí el vínculo entre libertad y responsabilidad que analiza Sen (2000). La libertad individual, en tanto capacidad de perseguir la vida que el individuo posee razones para valorar, tiene una relación de interdependencia con la responsabilidad. La misma tiene una doble dirección:

Sin la libertad sustantiva y la capacidad de hacer algo, una persona no puede ser responsable de hacerlo. Pero en realidad tener la libertad y la capacidad de hacer algo impone a la persona el deber de considerar si hacerlo o no, y esto implica responsabilidad individual. En este sentido, la libertad es tanto necesaria como suficiente para la responsabilidad (Sen, 2000, p.344)6.

Así, una adecuada comprensión de la educación desde el enfoque de las capacidades descansa sobre la agencia individual, definida en términos de la explicación anteriormente dada. Desde esta perspectiva, el sistema educativo no puede comprender a los educandos como meros recipientes de contenidos y conocimiento, que resultan beneficiados del mismo para maximizar sus oportunidades futuras de desarrollo. Tal visión resultaría, a nuestro entender, insuficiente, superflua y estéril para pensar el rol de la educación en el mundo actual. Más bien, se considera que los educandos deberían ser concebidos como individuos responsables, con las implicancias que conlleva la responsabilidad en términos de capacidad de elección, compromiso y libertad. Ellos mismos, en tanto seres humanos responsables, están a cargo de su propio proceso de aprendizaje y, asimismo, como seres reflexivos y

${ }^{6}$ La traducción es propia. 
pertenecientes a una comunidad (local, nacional y global), son responsables por las elecciones que realizan en relación con su entorno y los otros, en función de las situaciones de privación de capacidades y de falta de libertades que presencian cada día.

Respecto a tales nociones de la educación y los educandos, se retoma el concepto de educación para la libertad, propuesto por la filósofa norteamericana Martha Nussbaum (2009), quien ha colaborado estrechamente con Amartya Sen en el enfoque de las capacidades. En el presente estudio, se recupera dicha noción y las implicancias para la formación de los educandos, dejando de lado otras cuestiones analizadas por la filósofa en materia de educación en tanto no son pertinentes con el objeto del trabajo. Nussbaum (2009) construye el concepto educación para la libertad en contraste con el tipo de educación que ella percibe como dominante en el sistema universitario estadounidense, esto es la educación para el lucro o el enriquecimiento económico, basado en la inculcación de las habilidades básicas a una gran parte de los educandos y de habilidades técnicas avanzadas y especificas a algunos grupos de alumnos. La educación para la libertad trasciende el mero entrenamiento técnico y está orientado hacia la libertad de pensamiento, la capacidad crítica y el desarrollo humano entendido en términos de capacidades. La misma tiene por objetivo la formación de ciudadanos, comprendiendo la ciudadanía de forma amplia (esto es, cosmopolita, global) y densa (es decir, ligada a deberes y compromisos cívicos). No se trata, así, solamente de inculcar conocimientos técnicos, útiles para el mundo profesional, de manera unidireccional y predeterminada. Se trata, en cambio, de formar individuos para el desarrollo humano: en primer lugar, se trata de formar individuos que dispongan de las libertades y oportunidades necesarias para llevar a cabo la vida que tienen razones para valorar y, en segundo lugar, de promover el entendimiento de los objetivos de desarrollo humano para todos (y no sólo para sí mismos) como objetivos inherentes a la idea misma del ser humano y de una sociedad justa y decente. En este sentido, el fin de la educación debe ser, desde la óptica de Nussbaum (2008; 2009), el empoderamiento de los educandos para realizar elecciones políticas e impulsar la expansión de las capacidades para todos (Prudencio, 2009). Tal noción de educación parte necesariamente de "la idea de igual respeto para todos los seres humanos e igual derecho de todos a una gama de oportunidades humanas fundamentales" (Nussbaum, 2009, p.3).

Así entendida, la educación posee un componente profundamente igualitario, democrático y humanista. Se orienta hacia la justicia entendida como la ampliación de las capacidades, basada en la libertad individual y la responsabilidad cívica, buscando derribar los obstáculos y las privaciones a la libertad que se encuentran a nuestro alrededor. La noción de educación se aleja de las concepciones utilitaristas de la misma, deja de ser un mero componente funcional del mercado laboral, de los ecosistemas profesionales, del crecimiento 
económico (Nussbaum, 1992, 2008; 2009; Prudencio, 2009). Así entendida, la educación se vuelve sustantiva, se densifica, se hace abarcadora y portadora de libertades y responsabilidades. Toma un lugar fundamental en el mundo para la consecución del desarrollo humano inclusivo, otorgando a los sistemas educativos universitarios responsabilidades de una gran y profunda magnitud y a los educandos el papel central de agentes en su propio camino pedagógico y profesional.

\subsection{El enfoque de capacidades para la educación en Ingeniería: la metodología ensayada en la Universidad Católica de Córdoba}

¿Cómo poner en práctica en programas de educación un enfoque de este tipo para la educación en Ingeniería? El presente trabajo busca analizar, sin ánimos de hacerlo de manera acabada o sistemática, algunos rasgos del programa educativo desarrollado por la Facultad de Ingeniería de la Universidad Católica de Córdoba (UCC) para la carrera de Ingeniería en Computación. Los objetivos perseguidos aquí serán dos. Por un lado, se pretende identificar, en la práctica, algunos componentes del enfoque descripto anteriormente. Por el otro, el trabajo se propone encontrar, de manera por lo menos ilustrativa, mediante qué modos, abordajes e iniciativas se puede hacer operativa la educación para la libertad en la enseñanza específica de la Ingeniería. Si bien Nussbaum (2009) aplica tal noción concentrándose más bien en la enseñanza del arte y las humanidades, se considera que su aplicación a la educación en Ingeniería es de gran utilidad y necesidad en tanto ésta es una profesión que nos presenta grandes potencialidades, tanto como profundos desafíos hoy.

La Ingeniería se ha vuelto, en el curso de unas pocas décadas, una profesión fundamental para casi todo lo que el ser humano se propone hacer en el mundo profesional: todas las demás profesiones están hoy mediadas, de un modo u otro, por tecnología y por sistemas complejos, y, asimismo, la mayoría de las actividades humanas generan una gran cantidad de información que requiere ser analizada, procesada y comprendida para la resolución de problemas complejos. En su proceso de desarrollo y consolidación, la Ingeniería se fue compartimentando en diversos programas de estudio, que cobraron elevados niveles de especificidad y tecnicismo. Junto a ello, la Ingeniería se presenta hoy como una profesión altamente demandada, con fácil y directa salida a los mercados laborales dada la preparación especializada de los egresados. Siendo, la Ingeniería, un potente instrumento de cambio y de mejora de las condiciones de vida de los hombres y los sistemas humanos, nos parece fundamental orientar la enseñanza de la profesión en base a la educación para la libertad. Es evidente la necesidad de formar futuros ingenieros capaces de comprender los problemas actuales, libres de pensar de forma crítica, comprometidos con la 
expansión de las capacidades humanas para todos y responsables respecto a las propias elecciones profesionales.

El análisis propuesto del programa educativo en Ingeniería en Computación de la Universidad Católica de Córdoba, desde el enfoque de las capacidades, no pretende ser un estudio acabado o sistemático, sino más bien una serie de observaciones y reflexiones que pueden dar lugar, en el futuro, a ulteriores investigaciones en la materia. Asimismo, se trata de un ejercicio de tipo teórico, quedando fuera del objeto de estudio el análisis de los efectos o impactos que estas estrategias educativas tienen en el camino pedagógico de los educandos. Se considera el caso en dos partes: se analizan, en primer lugar, las competencias y el perfil del egresado en Ingeniería que la Universidad se propone fomentar $y$, en segundo lugar, el método de aprendizaje por proyectos con impacto social llevado adelante por los educadores de la Facultad de Ingeniería de la Universidad. De este modo, se busca primeramente comprender desde el enfoque de las capacidades los objetivos educativos de la Universidad en relación a las carreras de Ingeniería (entender el para qué de la enseñanza en Ingeniería) y, segundamente, presentar el método de aprendizaje por proyectos con impacto social como una innovadora metodología pedagógica (el cómo de la enseñanza en Ingeniería desde el enfoque de las capacidades).

\subsubsection{Las competencias del egresado en Ingeniería en Computación de la UCC}

El conjunto de conjunto de competencias que la Universidad entiende como deseables y necesarias para los egresados de la carrera de Ingeniería en Computación se componen de dos grupos: competencias genéricas, que serán el foco de nuestro análisis, y competencias específicas, que serán definidas sin mayor detenimiento en tanto quedan fuera del objeto de este análisis dado su tecnicismo. Las primeras son definidas por cada institución universitaria, que, en base a su marco institucional y al proyecto académico individual, determina para sus carreras, la estrategia pedagógica y los planes curriculares para asegurar competencias de egreso genéricas comunes a todas las carreras de ingeniería y necesarias para asegurar el perfil de egreso. Respecto a las competencias específicas ${ }^{7}$, el plan de estudios debe garantizar el desarrollo de las una serie de habilidades para las actividades reservadas definidas en la terminal (esto es, la especialidad de la carrera de Ingeniería) y verificar el cumplimiento, además, de la formación en el proyecto académico de la carrera, de

\footnotetext{
7 Para mayor detalle, las competencias específicas pueden ser consultadas en el documento citado, elaborado por la Asamblea del Consejo Federal de Decanos de Ingeniería de la República Argentina (2018).
} 
los alcances de título que defina la institución, con la profundidad y calidad propia de un título de ingeniero (CONFEDI $\left.{ }^{8}, 2018\right)$.

Las competencias genéricas establecidas para la carrera de Ingeniería en Computación se agrupan competencias tecnológicas, por un lado, y sociales, políticas y actitudinales, por el otro. En ambos grupos, se identifican una serie de competencias que estructuran el perfil del ingeniero en Computación que la Universidad se propone promover (CONFEDI, 2018). El esquema de competencias genéricas del egresado, en este caso, se conforma del siguiente modo (CONFEDI, 2018):

a. Competencias tecnológicas

1. Identificar, formular y resolver problemas de ingeniería.

2. Concebir, diseñar y desarrollar proyectos de ingeniería.

3. Gestionar, planificar, ejecutar y controlar proyectos de ingeniería.

4. Utilizar de manera efectiva las técnicas y herramientas de aplicación en la ingeniería.

5. Contribuir a la generación de desarrollos tecnológicos y/o innovaciones tecnológicas.

b. Competencias sociales, políticas y actitudinales

1. Desempeñarse de manera efectiva en equipos de trabajo.

2. Comunicarse con efectividad.

3. Actuar con ética, responsabilidad profesional y compromiso social, considerando el impacto económico, social y ambiental de su actividad en el contexto local y global.

4. Aprender en forma continua y autónoma.

5. Actuar con espíritu emprendedor.

Desde el enfoque de capacidades, se puede comprender que tal esquema de competencias está orientado a lo que Nussbaum (2008; 2009) llama educación para la libertad. Específicamente, se puede encontrar en el perfil del egresado de la carrera de Ingeniería en Computación detallado anteriormente, las habilidades que Nussbaum (2009) indica como fundamentales para la promoción de programas educativos basados en la educación para la libertad.

Nussbaum (2009) describe tres tipos de habilidades que una educación entendida desde ese enfoque ayuda a cultivar. En primer lugar, señala el pensamiento crítico como

${ }^{8}$ Siglas del Consejo Federal de Decanos de Ingeniería de la República Argentina. 
habilidad central, entendiendo al mismo en términos socráticos, esto es como reflexión crítica de nosotros mismos y nuestras propias tradiciones, como la capacidad de examinarnos a nosotros mismos y pensar acerca de las razones que nos llevan a inclinarnos por aquello que elegimos. Indica que el mismo es fundamental para la vida en democracia y, más aún, para la solidez de nuestras democracias actuales, caracterizadas por sociedades plurales y diversas donde se requiere que los ciudadanos sean capaces de entablar diálogos interculturales profundos (Nussbaum, 2008; 2009) A ello, específicamente pensando desde América Latina, nos resulta imperioso recalcar la necesidad de nuestras sociedades de recuperar los lazos sociales entre grupos socio-económicos diversos con el objetivo de promover el reconocimiento de alteridades (no sólo relacionadas a lo cultural, sino también lo étnico, lo social y lo económico) en el seno de nuestras comunidades y, partiendo del pensamiento crítico y el diálogo, buscar consolidar sistemas más justos e inclusivos. Nussbaum (2009) sostiene que, más que parte de la currícula, el pensamiento crítico debe ser un componente del espíritu de la pedagogía de una institución educativa y su puesta en práctica consiste en que cada educando sea tratado como un individuo en pleno proceso de desarrollo, cuyos aportes a la discusión en clase y al espacio educativo son válidos, activos y creativos. Comprender, así, el rol activo que tienen los educandos, sus voces y sus acciones en el contexto educativo es central para que la institución promueva una educación orientada hacia la libertad. Se entiende que esta habilidad puede encontrarse en el conjunto de competencias sociales, políticas y actitudinales establecido por la UCC para el egresado de Ingeniería en Computación y que la misma se constituye como la meta buscada a través del establecimiento de tales competencias y de las acciones emprendidas al respecto.

En línea con ello, la segunda habilidad que propone Nussbaum (2009) es la capacidad de concebirnos a nosotros mismos como miembros de una Nación y un mundo heterogéneos, donde existen diferencias de poder y de oportunidades. Promoviendo esta habilidad e impulsando a los educandos a llevarla a la acción en la búsqueda de un mundo más justo, la UCC ha estipulado la tercera competencia social, política y actitudinal en estos términos y, a partir de ella, promueve la inclusión de contenidos curriculares en el plan de estudios de Ingeniería en Computación, así como una serie de actividades de Responsabilidad Social Universitaria. Con ello, se persigue el doble objetivo de que los educandos alcancen un entendimiento integral y abarcador de los procesos sociales, históricos y económicos, comprendiendo y acercándose a las alteridades sociales, económicas e históricas que encuentran; y que pongan en práctica modos de buscar el desarrollo humano de los otros a la vez que el propio, entendido como ampliación de las oportunidades de perseguir una vida plena (Nussbaum, 2009). 
En tercer lugar, Nussbaum (2009) señala como habilidad fundamental también la imaginación narrativa. Con tal término, se refiere a la capacidad de separarse de la propia situación existencial para pensarse en el lugar de Otro, sabiendo leer e interpretar la historia de éste, sus emociones y deseos. Las demás habilidades pueden caer en visiones obtusas o egocéntricas si no son cultivadas desde la simpatía, entendida desde su significado etimológico como el acto de sentir igual que otro (del griego sympátheia, syn - convergencia - y pathos - sufrimiento, afección). Nussbaum (2009) indica que comprender la alteridad desde ese lugar y ver a los demás seres humanos como personas plenas (como un quién y no como un qué) no es innato en nosotros, sino que debe ser promovido y puesto en práctica por una educación que ponga en movimiento la imaginación respecto al otro, y que, desde la misma, impulse a los educandos a alcanzar una profunda comprensión de la experiencia de otro grupo u otra persona. Entre las competencias sociales, políticas y actitudinales propuestas por la UCC se encuentra este objetivo, que se materializa en la escucha activa, la comprensión del mundo y el compromiso social. Este objetivo se busca alcanzar con la inclusión de contenidos curriculares específicos en los planes de estudio, junto con la visibilización de situaciones y problemáticas específicas que interpelan a los estudiantes tanto desde la teoría como desde la práctica.

\subsubsection{El método de aprendizaje por proyectos con impacto social}

Las habilidades descriptas por Nussbaum (2009) pueden ser rastreadas en la estructura de competencias diseñada para el perfil del egresado en Ingeniería en Computación específicamente entre las competencias sociales, políticas y actitudinales, como ha sido descripto anteriormente. Cabe preguntarse, a partir de ello, de qué modo las instituciones educativas pueden fomentar tales habilidades, que son el núcleo de la educación para la libertad, más allá de incluir contenidos curriculares en los planes de estudio (como materias de formación filosófica, sociológica, etc.) y de las actividades de voluntariados e intervención social anteriormente mencionadas. Específicamente, cabe preguntarse de qué modo pueden ponerse en práctica estrategias pedagógicas para fomentar las habilidades propias de la educación hacia la libertad. Se observa, en el programa educativo de la carrera de Ingeniería en Computación, una forma innovadora de cultivar tales habilidades: el método de aprendizaje por proyectos con impacto social.

Cada año, en una clase de la carrera de Ingeniería en Computación, los educandos emprenden la realización de un proyecto o de un trabajo de investigación de principio a fin. Se requiere a los educandos que en el mismo se integren las distintas áreas temáticas de la carrera, de modo que se generen procesos de interacción entre las competencias específicas cultivadas por el plan de estudios y, asimismo, buscando desarrollar la capacidad de 
observación y de identificación de condiciones de contorno del problema objeto del trabajo. Debe contemplar el diseño, desarrollo y/o implementación del mismo y se deben abordar casos concretos induciendo el análisis crítico y la actividad creativa. Para el tratamiento del problema de estudio, se solicita ajustar la solución a las necesidades del medio y aplicar la tecnología disponible o a desarrollar (UCC, 2016). Por último, se requiere que las estrategias y respuestas impliquen (UCC, 2016):

- Propuestas relativas a problemáticas prioritarias para nuestra sociedad, especialmente concernientes a los sectores sociales más vulnerables.

- Respuestas concretas a la problemática analizada, altamente viables de ser implementadas y escaladas, con alto potencial de impacto social.

- Consolidación de enfoques integrales e interdisciplinares, con abordajes complejos a la problemática tratada.

- Participación, involucramiento y articulación con otros actores (instituciones, organizaciones sociales, etc.) provenientes de diversos niveles (barrial, municipal, provincial, regional, nacional o internacional), de modo de fortalecer la relación entre la academia y el medio, abriendo nuevos espacios de colaboración y trabajo conjunto.

A modo de ejemplo, se describen brevemente cuatro proyectos que han sido realizados en los últimos años: dos de ellos consisten en soluciones tecnológicas orientadas a la salud y los otros dos en soluciones educativas. En primer lugar, mediante un proceso de diagnóstico y trabajo conjunto con la Clínica Universitaria Reina Fabiola, se observó la necesidad de disponer de herramientas tecnológicas que permitan realizar un seguimiento de los pacientes que sufren migrañas e identificar una serie de factores específicos para cada uno con el objetivo de prescribir tratamientos más efectivos. Así, se desarrolló una "aplicación móvil capaz de recabar un conjunto de información relativa a crisis de pacientes migrañosos con el objetivo de mejorar el tratamiento y la calidad de vida de los mismos" (Pautasso et al. 2016, p.1).

En segundo lugar, otro proyecto que se enfocó en desarrollar una solución tecnológica aplicada a la salud es el de "ACUFEST", una aplicación móvil para pacientes con acúfenos, es decir pacientes que perciben un sonido en ausencia de una estimulación exterior. También en este caso, el desarrollo del proyecto fue realizado en conjunto con el equipo de trabajo de la Clínica Universitaria Reina Fabiola, junto a quienes los estudiantes realizaron el diagnóstico de la necesidad y el diseño de la aplicación en función de los requerimientos médicos identificados. La solución desarrollada tiene el objetivo de "analizar, distinguir y evaluar el acúfeno y generar estímulos sonoros capaces de inhibirlo" (Colombo et al. 2019). 
En tercer lugar, se destaca un proyecto que consiste en una solución tecnológica aplicada al ámbito educativo para niños con discapacidades. Los estudiantes, en este caso, identificaron que hay una tendencia creciente a utilizar las TIC como herramientas de apoyo en la educación de niños con discapacidad y que hay experiencias de impacto positivo en el uso de entornos lúdicos para mejorar las habilidades cognitivas y otras destrezas en la educación de personas con distintos tipos de discapacidad. La solución consiste en "la implementación de un entorno lúdico desarrollado para un centro educativo terapéutico" donde asisten niños con distintos tipos de discapacidad (Castagnola et al. 2015, p. 1).

Por último, se destaca otro proyecto que aplica la tecnología a la mejora de los procesos de aprendizaje. En este caso, el trabajo se centra en el desarrollo de un "videojuego educativo de rol online, que permite a los alumnos aprender jugando" (Marucci et al. 2015, p. 1). La elección del uso y desarrollo de un videojuego con fines pedagógicos se justifica en tanto se considera al juego como una "una poderosa herramienta que favorece la sociabilidad, permite desarrollar la capacidad creativa, crítica y comunicativa de las personas" (Marucci et al., 2015, p. 2). Se indica, además, que éste estimula habilidades como la acción, la reflexión, la expresión, la imaginación, el razonamiento, la observación, la asociación y la comparación (Marucci et al. 2015).

Reflexionando acerca de los proyectos brevemente detallados y los requerimientos formales descriptos anteriormente, desde el enfoque de capacidades y la propuesta teóricoeducativa de Martha Nussbaum (1992; 2008; 2009; Prudencio, 2009; Sen, 2000), se observa que el método de aprendizaje por proyectos de impacto social es una sólida estrategia pedagógica para la promoción de la educación para la libertad. La estrategia se basa en una premisa que resulta fundamental: los educandos son los agentes principales en su propio proceso de aprendizaje. Tal premisa es, en este caso, no sólo un postulado teórico desde donde se diseña el plan curricular, sino más que nada un principio que regula el modo mismo en que se proponen los contenidos del plan. Los educandos son responsables de todo el proceso que conlleva la realización de proyecto o trabajo de investigación, ellos mismos proponen la problemática a tratar a partir del análisis de la realidad, un calendario de actividades y un diseño de proyecto, así como ellos mismos son responsables de la implementación, la articulación con otras instituciones y actores sociales, etc. El papel de los educadores consiste en realizar el acompañamiento, brindando asesoramiento y recomendaciones si son requeridas por los educandos, y en la revisión final para la calificación de los proyectos realizados.

De este modo, el método de aprendizaje por proyectos con impacto social cultiva las competencias del perfil del egresado, detalladas anteriormente, desde la base del 
reconocimiento de la agencia, concepto central para el enfoque de las capacidades (Nussbaum, 2009; Sen, 2000). Los educandos, concebidos como agentes y no como meros recipientes de contenidos curriculares, cultivan un tipo de educación que va más allá del aprendizaje de una disciplina técnica, que se funda sobre la comprensión del mundo que los rodea, la identificación de grupos y sujetos como otros en tanto personas plenas en su derecho a una vida digna de ser vivida y la interpelación crítica acerca de situaciones de privación de capacidades para el desarrollo de aquellos otros.

Con ello, esta metodología fomenta la puesta en práctica de la responsabilidad, entendida en términos de Amartya Sen (2000). Los educandos se enfrentan con la libertad de escoger una problemática a tratar, observando el contexto social y comunitario cercano. Junto a tal libertad, se les presenta la responsabilidad de dar respuestas válidas, con elevados niveles de impacto social, factibles dados los recursos humanos, de tiempo y tecnológicos disponibles y viables en el contexto del problema identificado. Así, en el proceso de diseño e implementación de los proyectos, los educandos viven un proceso de puesta en práctica de la libertad ligada a la responsabilidad de escoger y de actuar en consecuencia. Un ejercicio de tal magnitud tiene un rol fundamental en la construcción y la formación de los valores y la ética basados en la educación para la libertad.

Partiendo desde la base de la agencia, la libertad y la responsabilidad, el método de aprendizaje por proyectos con impacto social impulsa a los educandos a cultivar las tres habilidades propuestas como deseables por Martha Nussbaum (2009), esto es: el pensamiento crítico, la capacidad de concebirnos a nosotros mismos como miembros de una Nación y un mundo heterogéneos y la imaginación narrativa. Asimismo, esta metodología pedagógica consolida la aprehensión de las competencias detalladas en el perfil del egresado de la carrera de Ingeniería en Computación, tanto genéricas como específicas, estimulando una interacción compleja entre las competencias tecnológicas y aquellas sociales, políticas y actitudinales para la definición de la problemática social a tratar y la articulación con actores e instituciones diversos a la Universidad, así como también una interacción entre las competencias tecnológicas específicas entre sí para el diseño de la solución tecnológica, su desarrollo e implementación a partir de la puesta en práctica de conocimientos pertenecientes a diversas áreas temáticas del plan de estudios.

\section{Conclusión}

El escenario actual presenta una alta complejidad global, caracterizado por la volatilidad, la existencia de amplias desigualdades y brechas socioeconómicas, grandes potencialidades en materia científica y tecnológica, inminentes riesgos globales (como el 
cambio climático, la inestabilidad nuclear, la seguridad energética, etc.) y un grado de interconexión entre actores, variables y zonas geográficas nunca antes imaginado. Este escenario se complejiza aún más si se observan las especificidades de nuestra región. América Latina vive hoy procesos de crecimiento y, a la vez, inestabilidad política, se enfrenta hace décadas al desafío de consolidar el camino al desarrollo inclusivo, emerge como continente con grande potencial en términos de recursos naturales, energéticos y humanos, etc. En un contexto tal, el rol de la tecnología es central en el impulso de ciertas tendencias que marcan el rumbo de las sociedades: hasta ahora, ¿la tecnología ha promovido un desarrollo inclusivo? ¿O ha, más bien, contribuido a expandir las desigualdades económicas y tecnológicas entre países y dentro de ellos? ¿Está a nuestro alcance proponer y poner en práctica un nuevo rol para el avance tecnológico? Con ello, ¿puede la Ingeniería constituirse en una profesión que minimice las desigualdades e impulse un mayor acceso a oportunidades equitativas?, ¿puede llegar de modo cada vez más equitativo y ubicuo a las sociedades y los individuos?, ¿puede defender el acceso inclusivo al conocimiento?

Para abordar estas preguntas y en miras a defender un nuevo rol de la Ingeniería, se retoma el concepto de desarrollo de Amartya Sen (2000; 2012), se analiza su teoría del desarrollo basado en las capacidades y el concepto propuesto por Martha Nussbaum (2009) de educación para la libertad. La educación, entendida como educación para la libertad, trasciende el mero entrenamiento técnico, está basada en el pensamiento crítico, la imaginación narrativa y la comprensión acabada del mundo que nos rodea y se orienta al desarrollo humano entendido en términos de capacidades. La misma posee un rol fundamental en la expansión del desarrollo humano en tanto su objetivo es formar individuos que, en primer lugar, dispongan de las libertades y oportunidades necesarias para llevar a cabo la vida que tienen razones para valorar y, en segundo lugar, pongan en práctica la promoción y expansión del desarrollo para todos (y no sólo para sí mismos). La educación para la libertad se funda en el reconocimiento de la agencia de los educandos, reivindica la libertad de los mismos como sujetos plenos y promueve la responsabilidad en relación al mundo y los otros (Nussbaum, 2009).

Convencidos de que todo ejercicio teórico es, además, un compromiso intelectual y práctico con el mundo observado, resulta fundamental hacer dialogar las categorías analizadas con las realidades educativas de nuestro país y nuestra región. Es por ello que, con ánimos de abrir interrogantes para futuras investigaciones, se intenta aquí un sencillo análisis de algunos rasgos del programa educativo de Ingeniería en Computación de la Universidad Católica de Córdoba con el objetivo de identificar, en la práctica, algunos componentes del enfoque adoptado. Específicamente, en primer lugar, se analizan las competencias establecidas para el perfil del egresado en Ingeniería que la Universidad se 
propone formar buscando comprender, en la práctica, desde el enfoque de las capacidades los objetivos educativos de la Universidad en relación a las carreras de Ingeniería (entender el para qué de la enseñanza en Ingeniería). En segundo lugar, se estudia el método de aprendizaje por proyectos con impacto social desarrollado por la Facultad de Ingeniería, intentando rastrear estrategias y abordajes innovadores, de manera por lo menos ilustrativa, por los que puede hacerse operativa la educación para la libertad en la enseñanza específica de la Ingeniería (el cómo de la enseñanza en Ingeniería).

Esta tarea, de lectura teórica y observación, resulta fundamental y necesaria para el objeto del presente trabajo: la educación en Ingeniería en el mundo de hoy. El enfoque de las capacidades, aplicado a la temática educativa, se constituye como una innovadora manera de analizar las problemáticas que conciernen a la materia en el contexto actual, implicando un abordaje con un gran potencial para la puesta en práctica de nuevas estrategias educativas. En este sentido, se abre un espacio para futuras líneas de investigación teórica y propuesta práctica con el objetivo de orientar la educación en Ingeniería a la expansión del desarrollo humano y la reducción de las apremiantes desigualdades sociales, económicas y tecnológicas de nuestra región. Una educación de tal magnitud necesariamente estará fundada en la capacidad de agencia de los educandos y será puesta en marcha por la libertad.

\section{Referencias bibliográficas}

Acemoğlu, D. y Robinson, J. (2012). Why Nations Fail: The Origins of Power, Prosperity, and Poverty. Crown Publishing Group. Edición Kindle.

Nussbaum, M. C. (1992). Human Functioning and Social Justice: In Defence of Aristotelian Essentialism. Political Theory, 20, 202-246.

Nussbaum, M. (2002). Las mujeres y el desarrollo humano. Barcelona: Herder.

Nussbaum, M. C. (2008). Liberty of conscience: In defense of America's tradition of religious equality. New York: Basic Books.

Nussbaum, M. C. (2009). Education for Profit, Education for Freedom. Liberal Education, 95(3). Recuperado de: https://eric.ed.gov/?id=EJ861161.

O'Donnell, G. (2010). Democracy, Agency, and the State. Theory with comparative intent. Serie Oxford Studies in democratization, Editor Laurence Whitehead. Nueva York: Oxford University Press,

O’Donnell, G. (2011). Modernización y autoritarismo. Buenos Aires: Prometeo Libros.

Prudencio, J. J. B. (2009). Martha Nussbaum, Peter Euben y la educación socrática para la ciudadanía. Revista de educación, 350. Septiembre-diciembre 401-422. 
Recuperado de: http://www.revistaeducacion.educacion.es/re350/re350_17.pdf.

[Consultado por última vez el 13 de octubre de 2019].

Sen, A. (1992) Inequality Reexamined. United States : Oxford University Press.

Sen, A. (2000). Development as freedom. Nueva York: Anchor Books. Versión Kindle.

Sen, A. (2012). La idea de la justicia. Buenos Aires: Taurus.

Urquijo, M. (2014). La teoría de las capacidades en Amartya Sen. Revista EDETANIA, (46), 63-80.

\section{Otros documentos consultados}

Asamblea del Consejo Federal de Decanos de Ingeniería de la República Argentina, CONFEDI (2018). Propuesta de estándares de segunda generación para la acreditación de carreras de Ingeniería en la República Argentina. "Libro Rojo De Confedi".

Colombo, M.; Bosio, M. A.; Vigliano, M.; Chiodi, G. (2019) ACUFTEST, aplicación móvil para pacientes con acúfenos. Paper presentado en Séptimo Congreso Nacional de Ingeniería Informática - Sistemas de Información, DIIT Universidad Nacional de La Matanza - Red IISIC. Disponible en https://conaiisi2019.unlam.edu.ar/pdf/2019-CONAIISI-MEMORIAS-7MAEDICION.zip.

Castagnola, M. E., Bosio, M. A., \& Chiodi, G. A. (2015). Juegos serios aplicados a niños con discapacidades. En II Simposio Argentino sobre Tecnología y Sociedad (STS)JAIIO 44 (Rosario, 2015). Disponible en: http://44jaiio.sadio.org.ar/sites/default/files/sts36-43.pdf.

Marcucci, M., Aliciardi, A. E., Collino, F., Cunto, G. D., Bosio, M. A., \& Chiodi, G. A. (2015). Videojuegos como parte del aprendizaje. En II Simposio Argentino sobre Tecnología y Sociedad (STS)-JAllO 44 (Rosario, 2015). Disponible en http://44jaiio.sadio.org.ar/sites/default/files/sts36-43.pdf.U

Universidad Católica de Córdoba, UCC (2016). Trabajo Final de Grado. Reglamentación y Documentación. Anexo de Resolución de Decanato. 